\title{
A Monolithic Buck Converter With Near-Optimum Reference Tracking Response Using Adaptive-Output-Feedback
}

\author{
Patrick Y. Wu and Philip K. T. Mok, Senior Member, IEEE
}

\begin{abstract}
A monolithic output-ripple-based buck converter with adaptive output and ultra-fast reference tracking is presented. Fixed-switching-frequency $\mathrm{V}^{2}$-control is used in steady-state operation; while its speed limitation during reference tracking is eliminated by employing end-point prediction, a novel oscillator with clock-holding function, and the proposed adaptive-outputfeedback (AOFB)-scheme. The AOFB-scheme is obtained from a detailed analysis of the dynamics of general output-ripple-based controller during reference tracking. Then, an adaptive feedback voltage which satisfies the switching criterion for optimum switch control in reference tracking is formulated, and is incorporated into the controller design. The resultant single ON/OFF-switching during reference tracking is very close to the optimal solution for fast reference tracking. Moreover, it also leads to the lowest switching loss and minimized conduction loss at the fastest tracking speed, making the converter very suitable for adaptiveoutput applications.

Fabricated in a $0.35 \mu \mathrm{m}$ CMOS process, the $3-\mathrm{V}$ input prototype chip exhibits stable operation across a wide output range of 0.7-2.4 V. High tracking speed within $10 \mu \mathrm{s} / \mathrm{V}$ is measured with different output voltages and output voltage steps. Maximum efficiency of $96 \%$ is obtained at $260 \mathrm{~mA}$ output current, while maximum output current is $800 \mathrm{~mA}$.
\end{abstract}

Index Terms-Dynamic voltage scaling, end-point prediction, output-ripple-based control, reference tracking, $\mathrm{V}^{2}$-control.

\section{INTRODUCTION}

$\mathbf{T}$ HE prevalence of battery-operated portable devices like cellular phones and media players creates a great demand for high-quality switching DC-DC converters. The desirable qualities generally include high efficiency, small volume, tight static and dynamic regulations, and low EMI noise. Regarding efficiency, a recent low-power VLSI technique namely dynamic voltage scaled (DVS) calls for a new type of "adaptive power supply" [1]-[5], which requires its output voltage to track the reference voltage when the latter has a step change. As a result, two new parameters, namely transition (tracking) time and transition energy, are introduced to characterize the power supply's performance on speed and energy conservation during reference tracking [4]. Obviously, too long transition time may reduce energy saving of a DVS system or adds considerable latency to resume high-speed operation. Meanwhile, too large

Manuscript received January 29, 2007; revised June 29, 2007. This work was supported in part by the Research Grant Council of Hong Kong SAR Government under Project 617705.

The authors are with the Department of Electronic and Computer Engineering, The Hong Kong University of Science and Technology, Kowloon, Hong Kong (e-mail: patwu@ust.hk; eemok@ece.ust.hk).

Digital Object Identifier 10.1109/JSSC.2007.907169 transition energy adds significant overhead to the energy saving by DVS.

Unfortunately, few literatures discuss the performance of switching converters (using different control methodologies) applied in a DVS system in detail. There are some recent works [6], [7] discussing the design of controller for DVS; but all of them are digital controllers, which usually involves large and sophisticated look-up tables and digital structures typically not familiar to analog circuit designers. On the other hand, outputripple-based control, e.g., hysteretic and $\mathrm{V}^{2}$ [8]-[10], which exhibits the fastest load transient response among common analog controls, remains rarely explored regarding to their performance in a DVS system. This paper aims to bridge this gap by considering a $\mathrm{V}^{2}$-controlled buck converter. $\mathrm{V}^{2}$-control is chosen because of its tight regulation and fixed switching frequency [9], [10]. After a close examination of its circuit structure and the ideal dynamics during reference tracking, the conventional $\mathrm{V}^{2}$-control is found to have poor transition time. Hence, a buck converter based on a modified $\mathrm{V}^{2}$-control is proposed in this paper. By using the end-point prediction (EPP) scheme [11], a novel oscillator with clock-holding function, and the proposed adaptive-output-feedback (AOFB) technique, the proposed buck converter achieves an ultra-fast reference tracking speed for a wide range of reference transition scenarios under different output voltages and voltage steps. Not only does the converter achieve the fastest tracking time, but also has the two unique characteristics during reference tracking: absence of overshoot/ undershoot in output voltage and inductor current, and minimum number of switching activities (single ON/OFF-switching). These features reduce the conduction loss and minimize the switching loss during tracking period, thus transition energy is also considerably conserved under the condition of fastest transition time.

This paper is organized as follows. Section II reviews the conventional fixed-frequency $\mathrm{V}^{2}$-controlled buck converter and its limitation. Section III presents the improved reference tracking performance using the EPP scheme and its pitfall. Section IV analyzes the dynamics during reference tracking, which leads to the need for a novel oscillator design and the proposed AOFB-scheme. Circuit implementation of major building blocks is discussed in Section V. Section VI presents the measurement results and compares with state-of-the-art adaptive DC-DC converters.

\section{REVIEW OF $\mathrm{V}^{2}$-ARCHITECTURE}

Fig. 1 shows the conventional fixed-frequency $\mathrm{V}^{2}$-architecture [9]. It has two distinct characteristics different from 


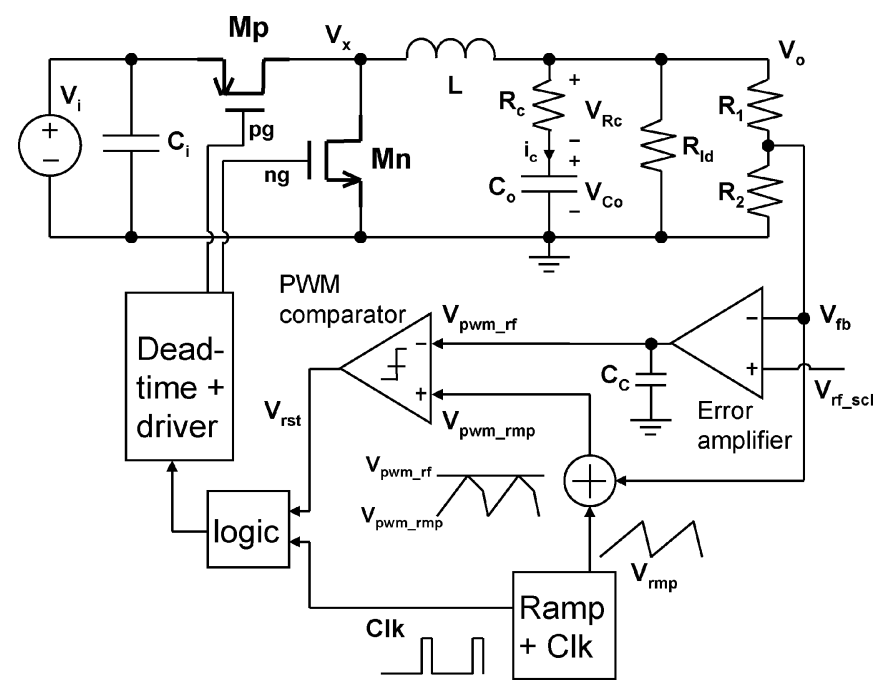

Fig. 1. Conventional $\mathrm{V}^{2}$-controlled buck converter.

typical voltage-mode or current-mode converters: 1) the existence of two feedback loops both from output voltage, $V_{\mathrm{o}}$, and $2)$ the origin of pulse-width modulated (PWM) ramp signal $\left(V_{\text {pwm_rmp }}\right)$. The slow feedback loop encompasses error amplifier, PWM comparator and power stage, while the fast feedback loop bypasses error amplifier by directly feeding $V_{\mathrm{fb}}$ (scaled $V_{\mathrm{o}}$ ) to the noninverting input of PWM comparator. The ramp signal is given by the output ripple due to the ESR of output capacitor $\left(R_{\mathrm{c}}\right)$. The artificial ramp $V_{\mathrm{rmp}}$ is only for avoiding sub-harmonic oscillation when duty-ratio is larger than $50 \%$. Since $V_{\text {pwm_rmp }}$ is mainly a scaled $V_{\mathrm{o}}$, a change in $V_{\mathrm{o}}$ due to load current step instantly causes a large change to its DC value, and thus instantly saturates the duty-ratio to $100 \%$ or $0 \%$. A fast load transient response is then obtained without a complex compensation network as required in typical voltage-mode or current-mode converters. In fact, the slow feedback loop is only intended for high static accuracy by using a high-gain error amplifier. Thus, a large compensation capacitor, $C_{\mathrm{c}}$, can be used for robust operation.

Yet, the considerations are different when reference tracking is concerned. When there is a step change in reference voltage, $V_{\mathrm{rf} \_s c l}$, the error amplifier has to drive the large $C_{\mathrm{c}}$ before change of duty-ratio is possible. This slow process usually lasts for more than $100 \mu \mathrm{s}$ when the change in $V_{\text {rf_scl }}$ is around hundreds of $\mathrm{mV}$ with a large $C_{\mathrm{c}}(>10 \mathrm{nF})$, hence forming the bottleneck of reference tracking speed.

\section{V V -ARCHITECTURE WITH EPP SCHEME}

To solve the problem of slow reference tracking in the dominant-pole compensated voltage-mode converter, an EPP scheme is proposed in [11]. The final error amplifier output voltage, $V_{\text {pwm_rf }}$, after reference transition is predicted by considering the geometric relationship between the duty-ratio, $V_{\text {pwm_rf }}$ and the upper and lower bounds of ramp signal. After a proper choice of the upper bound of ramp signal, EPP is done by adding $V_{\text {rf_scl }}$ to the inverting input of PWM comparator,

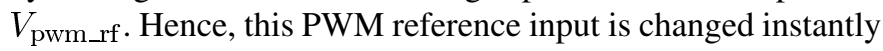
without requiring error amplifier's output to move significantly. The similar bypassing principle can be applied in $\mathrm{V}^{2}$-control

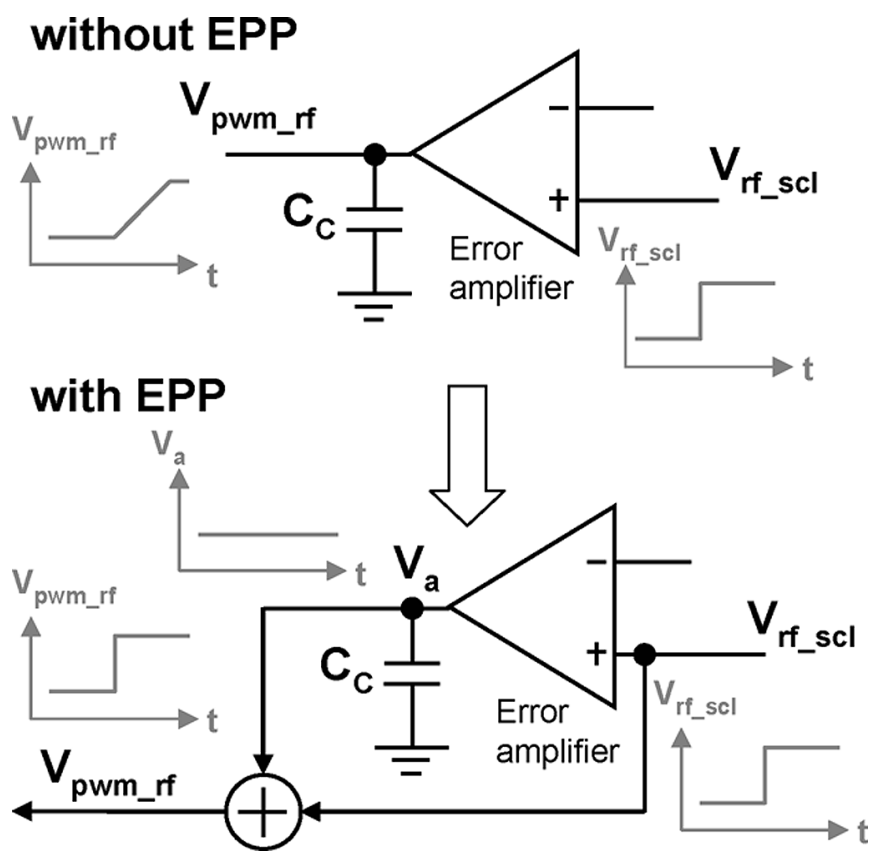

Fig. 2. Implementation of end-point prediction (EPP).

for reference tracking. Nonetheless, the prediction consideration is a bit different from that of voltage-mode control. Let us denote the desired $V_{\mathrm{o}}$ step as $\Delta V_{\mathrm{o}}$ and the corresponding changes in $V_{\text {rf_scl }}, V_{\mathrm{fb}}, V_{\text {pwm_rmp }}$, and $V_{\text {pwm_rf }}$ as $\Delta V_{\text {rf_scl }}$, $\Delta V_{\mathrm{fb}}, \Delta V_{\mathrm{pwm} \_\mathrm{rmp}}$, and $\Delta V_{\text {pwm_rf }}$, respectively. It can be easily proved that

$$
\Delta V_{\mathrm{rf} \_ \text {scl }}=b \Delta V_{\mathrm{o}}=\Delta V_{\mathrm{fb}}=\Delta V_{\mathrm{pwm} \_\mathrm{rmp}}
$$

where $b=R_{2} /\left(R_{1}+R_{2}\right)$ is the feedback factor given by the resistive divider. In steady-state, $V_{\text {pwm_rmp }}$ is very close to the dc-like $V_{\text {pwm_rf }}$ except an ac ripple of tens of $\mathrm{mV}$ (see Fig. 1). In reference tracking, such a small ripple is overwhelmed by the large $\Delta V_{\text {pwm_rmp }}(>300 \mathrm{mV})$. Therefore, we can conclude that the predicted $\Delta V_{\text {pwm_rf }}$ is roughly $\Delta V_{\text {rf_scl }}$ by (1). Hence, we simply add a reference feed-forward path to the PWM comparator as shown in Fig. 2 for end-point prediction. It is interesting to note that different ways of consideration leads to the same implementation in this work and the previous work [11].

The simulation result of reference down-tracking $\left(V_{\text {rf_scl }}\right.$ decrease) of $\mathrm{V}^{2}$-architecture with EPP is shown in Fig. 3. With $b=2 / 3, \Delta V_{\text {rf_scl }}=333.3 \mathrm{mV}, \Delta V_{\mathrm{o}}$ is expected to be $500 \mathrm{mV}$.

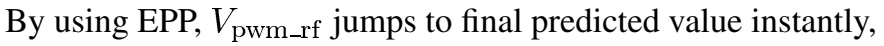
and $V_{\mathrm{o}}$ transition time is shortened to $15 \mu \mathrm{s}$ (a scaled $V_{\mathrm{o}}$ is shown in Fig. 3 for ease of axis scaling), when compared to hundreds of microseconds in the case without EPP. Yet, several adverse effects are observed. Firstly, there is an undershoot in $V_{\mathrm{o}}$. A brief explanation of the dynamics during reference tracking is useful for an initial understanding of the problem. The block "logic" in Fig. 1 is an SR-latch set by clock and reset by PWM comparator output, $V_{\text {rst }}$. When $V_{\text {rf_scl }}$ transits to lower value, $V_{\text {rst }}$ turns high to reset logic. $M_{n}$ then closes while $M_{p}$ opens to decrease inductor current, $i_{L}$. Since the logic is designed to be reset-dominant, $i_{L}$ will not increase (i.e., $\mathrm{M}_{\mathrm{n}}, \mathrm{M}_{\mathrm{p}}$ will not switch) until $V_{\text {pwm_rmp }}<V_{\text {pwm_rf }}$ and clock is issued. Fig. 3 shows the case 


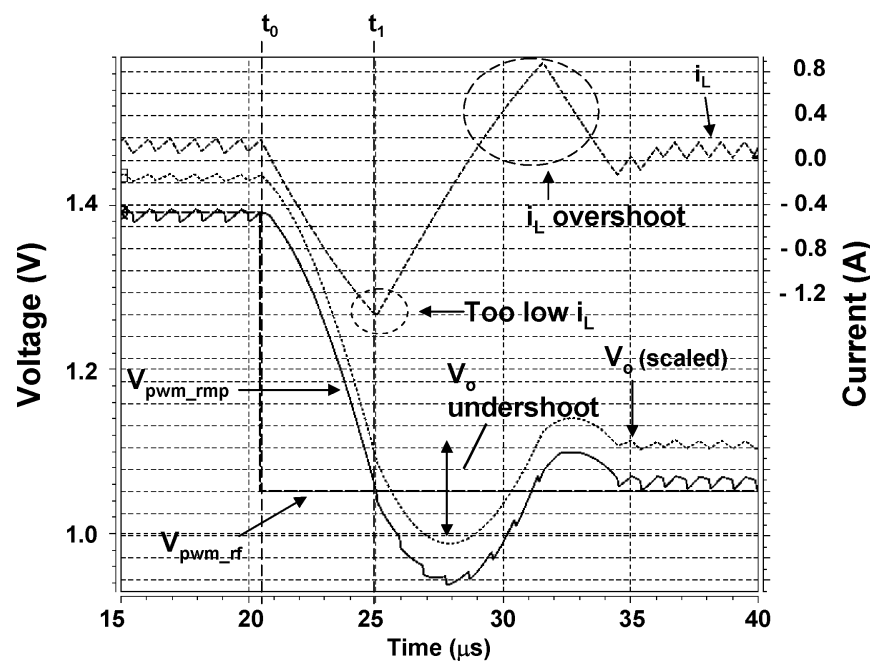

Fig. 3. Simulated reference down-tracking of $\mathrm{V}^{2}$ architecture with EPP.

when clock is issued as soon as $V_{\text {pwm_rmp }}<V_{\text {pwm_rf }}$ at $t_{1}$ (design of this clock circuit is covered in Section V). It can be concluded that end-point prediction speeds up tracking, but it does not guarantee proper reference tracking (e.g., absence of undershoot/ overshoot). Fig. 3 shows that direct comparison of PWM ramp signal and PWM reference signal is not a good criterion for determining switching activities during reference tracking. The dynamics behind reference tracking will be thoroughly analyzed in next section to formulate the criterion for proper reference tracking.

$V_{\mathrm{o}}$ undershoot during reference tracking should be avoided as it increases the transition time, and most importantly, affects the reliability of the digital circuits due to the lower supply voltage. Other adverse effects shown in Fig. 3 are too low $i_{L}$ at $t_{1}$ and the following $i_{L}$ overshoot for replenishing the lost charge in $C_{\mathrm{o}}$. Both of these effects increase conduction loss during transition and should be avoided.

\section{Proposed AOFB Scheme}

The model in Fig. 4 is used to derive the condition for optimum reference tracking in general output-ripple-based controller. Reference tracking starts at $t_{0}$ and ends at $t_{1}+t_{2}$ ( $t_{0}=0$ for easy discussion). Steady state current ripple in $i_{L}$ is neglected as it is small compared with the peak change of $i_{L}$ $\left(\Delta i_{L, \mathrm{rf}}\right)$ during reference tracking. Thus, the steady-state $V_{\mathrm{o}}$ ripple is also neglected. $V_{\mathrm{Co}}$ is the voltage across $C_{\mathrm{o}}$, which is $V_{\mathrm{o}}$ before reference tracking, and $V_{\mathrm{o}}-\Delta V_{\mathrm{o}}$ after reference tracking. Assuming load current $i_{\mathrm{ld}}$ is unchanged, $i_{L}$ equals $i_{\text {ld }}$ before and after reference tracking. The optimum reference tracking happens when $i_{L}$ experiences a straight decrease from $t_{0}$ to $t_{1}$ (period A), followed by a straight increase from $t_{1}$ to $t_{1}+t_{2}$ (period B) back to its original level, such that $V_{\mathrm{Co}}$ moves down by $\Delta V_{\mathrm{o}}$ at $t_{1}+t_{2}$ (single ON/OFF-switching) [6]. This is equivalent to using a near-triangular $i_{L}$ profile to remove the charge of $C \Delta V_{\mathrm{o}}$ in $t_{1}+t_{2}$ seconds. $i_{L}$ profile is not strictly triangular as the slope of $i_{L}$ in periods A and B are not constant but depends on a varying quantities $V_{\mathrm{o}}(t)$. However, for deriving an optimum reference tracking criterion that is useful for design purpose, the average slope in periods A and B can be defined as

$$
\begin{aligned}
-m_{2} & =\frac{-V_{\mathrm{o}, \mathrm{eff}}}{L} \\
m_{1} & =\frac{V_{i}-V_{\mathrm{o}, \mathrm{eff}}}{L}
\end{aligned}
$$

where $V_{\mathrm{o}, \text { eff }}$ is around the middle value between $V_{\mathrm{o}}$ and $V_{\mathrm{o}}$ $-\Delta V_{\mathrm{o}}$. Then, since the decrease of $i_{L}$ in period $\mathrm{A}$ is equal to the increase of $i_{L}$ in period $\mathrm{B}$, we have

$$
m_{2} t_{1}=m_{1} t_{2} .
$$

Making use of the fact that the shaded area represents the removed charge $C \Delta V_{\mathrm{o}}$, and (2)-(4), optimum time for switching power-MOSs $\left(t_{1}\right)$ can be derived as

$$
t_{1}=\sqrt{\frac{2 C_{\mathrm{o}} L(1-D) \Delta V_{\mathrm{o}}}{V_{\mathrm{o}, \mathrm{eff}}}}
$$

where $D=V_{\mathrm{o}, \text { eff }} / V_{i}$. It can be seen that the relation between $t_{1}$ and many quantities like $D$ and $\Delta V_{\mathrm{o}}$ is very sophisticated. There is no simple way to directly compute $t_{1}$ using simple circuits, unless a large look-up table implemented in digital FPGA (which is the approach used in previous works [6], [7]). Moreover, the previous work [6] did not implement a controller to close the loop for output regulation. Thus, the possibility of incorporating the above criterion into controller design needs to be investigated. Since the switching criterion in $\mathrm{V}^{2}$-control is $V_{\text {pwm_rmp }}<V_{\text {pwm_rf }}$, the change of $V_{\text {pwm_rmp }}$ at $t_{1}$ is derived as

$$
\begin{aligned}
\Delta V_{\mathrm{pwm} \_r m p}\left(\mathrm{t}_{1}\right) \\
\quad=b\left\{\Delta V_{\mathrm{Rc}}\left(t_{1}\right)+\Delta V_{\mathrm{Co}}\left(t_{1}\right)\right\} \\
\left.\quad=b\left\{R_{\mathrm{c}} m_{2} t_{1}+\frac{1}{C_{\mathrm{o}}} \int_{0}^{t_{1}}\left(m_{2} t\right) d t\right)\right\} \\
\quad=b\left\{R_{\mathrm{c}} \sqrt{2 C_{\mathrm{o}} \Delta V_{\mathrm{o}} m_{2}(1-D)}+(1-D) \Delta V_{\mathrm{o}}\right\}
\end{aligned}
$$

where (6c) is obtained by substituting (5) and (2). Given that $\Delta V_{\text {pwm_rf }} \approx b \Delta V_{\mathrm{o}}$, we propose to add the following adaptiveoutput-feedback-voltage to $V_{\text {pwm_rmp }}$ in period A:

$$
V_{\mathrm{aofb}}=-k \cdot D \Delta V_{\mathrm{o}}
$$

where

$$
k=b\left[1-R_{\mathrm{c}} \sqrt{\frac{2 C_{\mathrm{o}} m_{2}(1-D)}{D^{2} \Delta V_{\mathrm{o}}}}\right] .
$$

The effect is like adding an adaptive voltage $V_{\mathrm{a} o f b}$ to $V_{\mathrm{fb}}$ to meet switching criterion $\left(V_{\text {pwm }_{\text {rmp }}}<V_{\text {pwm_rf }}\right)$ at $t_{1}$. Fig. 5 illustrates this idea. At $t_{0}$ when $V_{\text {pwm_rf }}$ transits, $V_{\text {aofb }}$ is added to $V_{\text {pwm_rmp }}$, so that switching occurs at $t_{1}$ and $V_{\mathrm{o}}$ settles properly; otherwise, switching may happen at a later time $\left(t_{1}{ }^{\prime}\right)$ and $V_{\mathrm{o}}$ undershoot may occur. Finally, it should be noted that $V_{\text {aofb }}$ is removed from $V_{\text {pwm_rmp }}$ at $t_{1}+t_{2}$ when $i_{L}$ returns to steady-state level; otherwise, $V_{\text {pwm_rmp }}$ will experience an offset in steadystate. 


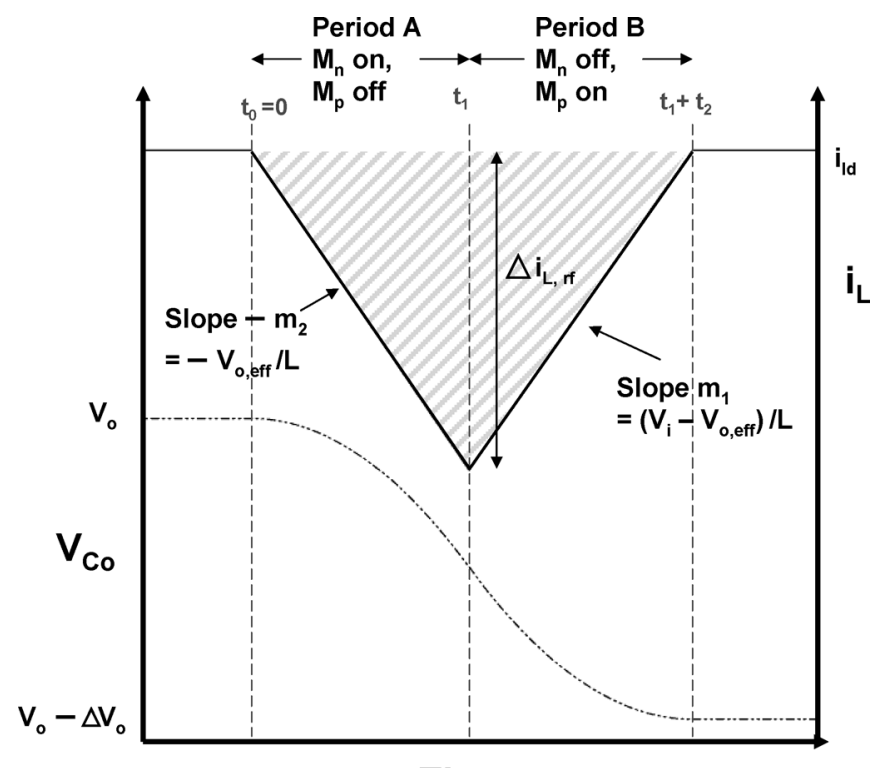

Time

Fig. 4. Model of optimum reference tracking.

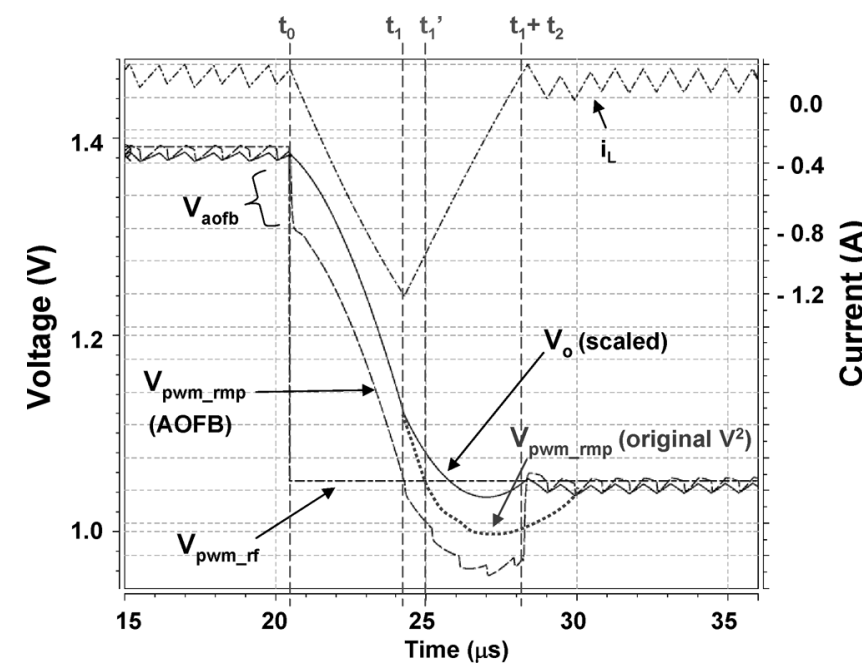

Fig. 5. Simulated optimum reference tracking with proposed AOFB technique.

Using a similar model, $V_{\text {aofb }}$ for reference up-tracking $\left(V_{\text {rf_scl }}\right.$ increase) is also derived and tabulated in Table I. It can be seen that $V_{\text {aofb }}$ is relatively simple if $k$ is close to a constant. Then, $V_{\text {aofb }}$ is only proportional to $D$ (or $1-D$ ) and $\Delta V_{\mathrm{o}}$ and it can be implemented in circuits. Fig. 6 shows the variation of $k$ against $D$ and $\Delta V_{\mathrm{o}}$. For $R_{\mathrm{c}}>0$, reference up- and down-tracking have different values of $k$, and $k$ varies with $D$ and $\Delta V_{\mathrm{o}}$. Yet, for practical applications we can select independent $k$-parameters for reference up- and down-tracking, and choose the largest $k$ required for the worst case (largest $\Delta V_{\mathrm{o}}$ ) so that for a given range of $V_{\mathrm{o}}$, no undershoot/ overshoot in $V_{\mathrm{o}}$ and $i_{L}$ will be observed. When the system asks for a smaller step size, the overly large $k$ will make the switching time a bit earlier than optimum and delay reference tracking a bit. Yet, the overall transient is not seriously affected, and the absence of undershoot /overshoot still results in energy saving. Comparing simulation results of Figs. 3
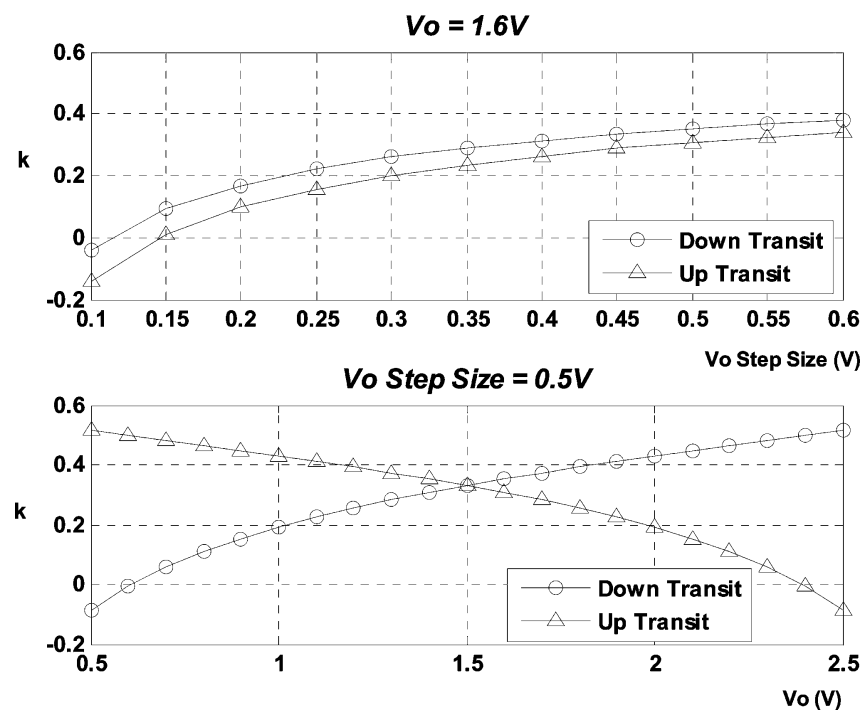

Fig. 6. Plot of $k$-parameter $\left(V_{i}=3 \mathrm{~V}, C_{\mathrm{o}}=10 \mu \mathrm{F}, L=4.7 \mu \mathrm{H}, R_{\mathrm{C}}=\right.$ $0.1 \Omega)$.

TABLE I

SUMMARY OF PARAMETERS OF AOFB TECHNIQUE

\begin{tabular}{|c|c|c|}
\hline Tracking Direction & Down & $\mathrm{Up}$ \\
\hline $\mathrm{V}_{\mathrm{aofb}}$ & $-k \cdot D \Delta \mathrm{V}_{\mathrm{o}}$ & $k \cdot(1-D) \Delta \mathrm{V}_{\mathrm{o}}$ \\
\hline $\mathrm{k}$ & $b\left[1-R_{c} \sqrt{\frac{2 C_{o} m_{2}(1-D)}{D^{2} \Delta \mathrm{V}_{\mathrm{o}}}}\right]$ & $b\left[1-R_{c} \sqrt{\frac{2 C_{o} m_{1} D}{(1-D)^{2} \Delta \mathrm{V}_{\mathrm{o}}}}\right]$ \\
\hline
\end{tabular}

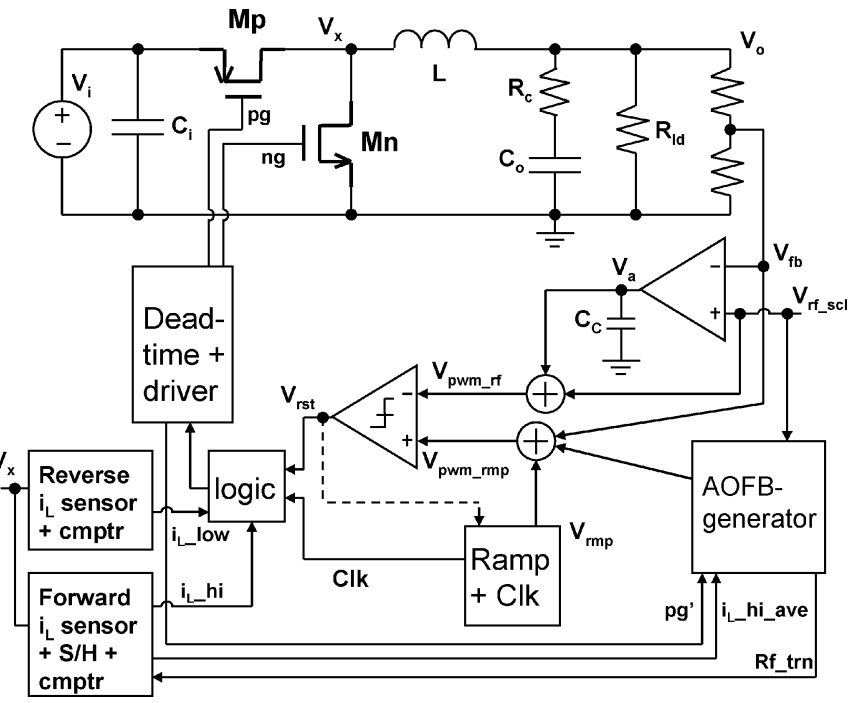

Fig. 7. Proposed $\mathrm{V}^{2}$-controlled buck converter with AOFB technique.

and 5 , conduction loss is found to be reduced by $50 \%$ with the absence of undershoot.

If the assumption of fixed $i_{\mathrm{ld}}$ is not true, forcing $i_{L}$ to return to original level as the criterion for removing $V_{\text {aofb }}$ is equivalent to having a little unbalance between load current and inductor current after reference tracking. This is similar to a slight load transient in nature, which can be promptly handled by $\mathrm{V}^{2}$-architecture without significantly affecting the settling time. 


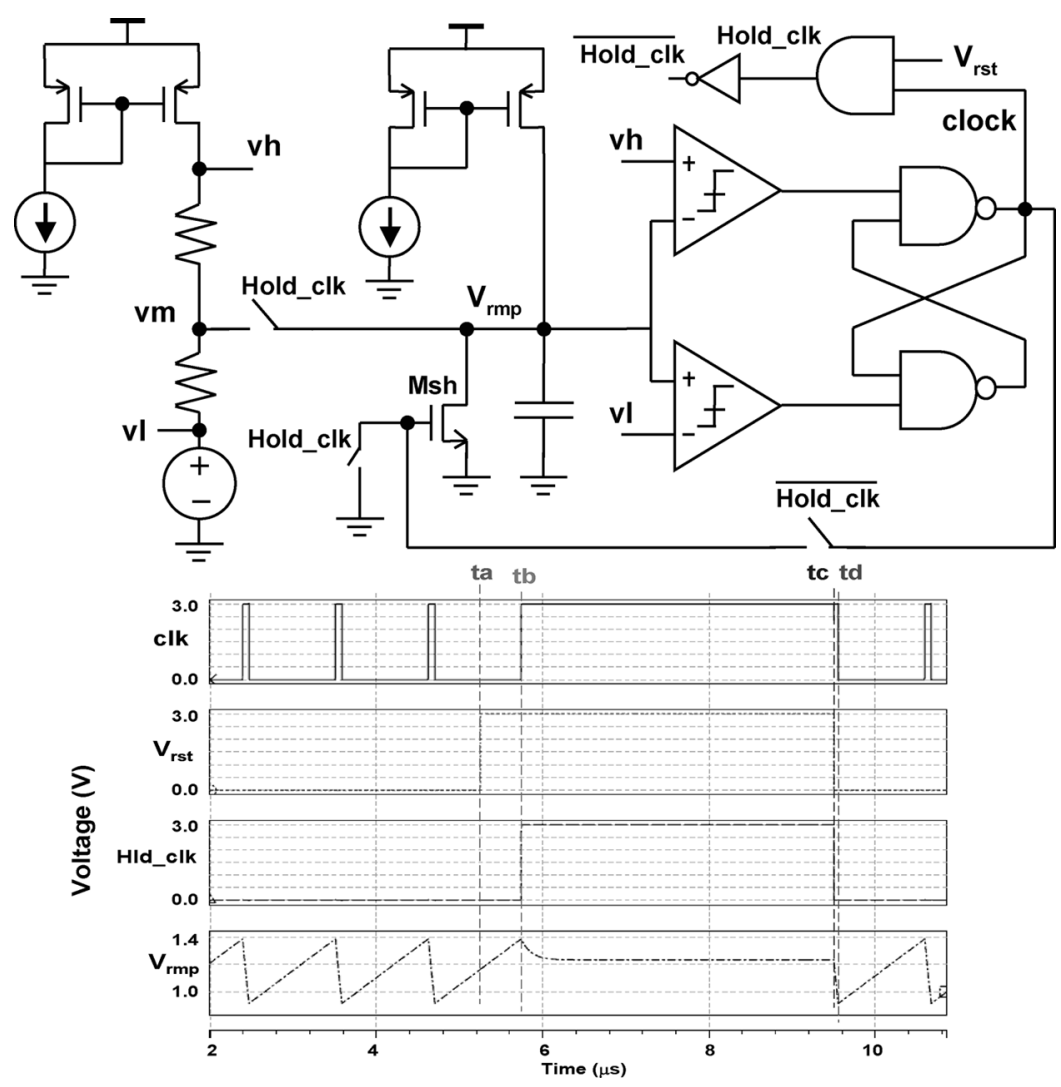

Fig. 8. Proposed oscillator with clock-holding function.

\section{Circuit ImPlementation}

Fig. 7 shows the proposed buck converter. EPP, a novel oscillator, and AOFB scheme are the new features. They are described in details in the following sections.

\section{A. Proposed Oscillator}

In the trailing-edge modulated converter, the logic is set by a periodic clock signal, while the reset input is the output of PWM comparator. In reference up-tracking, a reset signal can be issued instantly after the switching criterion is fulfilled. However, in reference down-tracking, the logic can only be set at the next clock following the optimum switching time. Hence, delay occurs. A novel oscillator with clock-holding function is thus proposed to optimize reference down-tracking.

Fig. 8 shows the proposed oscillator and its timing diagram. In steady-state, its operation is the same as a previous work [12]. Yet, during reference down-tracking, its clock can be held high until optimum switching time determined by AOFB-scheme. In the timing diagram, $V_{\text {rf_scl }_{\text {scl }}}$ down-transits at $t_{\mathrm{a}}$, so that PWM comparator output, $V_{\text {rst }}$, turns high. When the next clock pulse (clk) comes at $t_{b}$, Hld_clk turns high. Then the oscillator enters the "clock-holding" state by stopping the feedback of clk to $\mathrm{M}_{\mathrm{sh}}$, turning $\mathrm{M}_{\mathrm{sh}}$ off and clamping $V_{\mathrm{rmp}}$ to vm which is around middle of $\mathrm{vh}$ and $\mathrm{vl}$ (the upper and lower bounds of ramp signal). Hence, $V_{\text {rmp }}$ stays in between vh and vl, and keeps clk to stay high when Hld_clk is high. Due to the reset-dominant nature of the logic, the converter stays in reset state regardless of the clock. This clock-holding state lasts until $V_{\text {rst }}$ turns low at $t_{\mathrm{c}}$ (i.e., $V_{\mathrm{pwm}_{\mathrm{rmp}}}<V_{\text {pwm_rf }}$ ). Then, Hld_clk is cleared, and the oscillator resumes its operation, with $V_{\mathrm{rmp}}$ ramping down to $\mathrm{vl}$ and resetting clk at $t_{\mathrm{d}}$. Since clk remains high at $t_{\mathrm{c}}$ (and until $t_{\mathrm{d}}$ ) when optimum switching criterion is fulfilled, the logic is set instantly without the delay in conventional oscillator.

\section{B. AOFB Implementation}

Fig. 9 shows the main components inside AOFB-generator. Section IV showed that circuits extracting $V_{\text {rf_scl }}$ transition time, steady-state $i_{L}$, duty-ratio, and $V_{\text {rf_scl }}$ step size are required. They are implemented in simple circuits as described below.

$V_{\text {rf }}$-transition-detector comprises two comparators to detect the sudden transit of $V_{\text {rf_scl }}$ (i.e., start of reference tracking). Offset is built into the comparators to prevent false alarm. This detector is needed for an external analog $V_{\text {rf_scl }}$ signal and can be removed if the command of reference transition is provided by digital load. Two outputs of the detector, Rf_dn_dt and $\mathrm{Rf}_{-}$up_dt, trigger $\mathrm{T}_{\mathrm{Rf} \_ \text {trn }}$ logic to start the process of reference down- and up-tracking, respectively.

$\mathrm{T}_{\mathrm{Rf} \text { _trn }}$ logic marks the start and the end of the reference tracking. The process of reference tracking is signaled by the active high "Rf_trn" and is ended by inductor current $i_{L}$ returning to its original steady-state $\left(t_{1}+t_{2}\right.$ in Fig. 5). The time instant $t_{1}+$ $t_{2}$ is detected by the following mechanism: a sample-and-hold (S/H) following a forward inductor current sensor (shown in Fig. 7) periodically samples the inductor current value at steadystate. When Rf_trn turns high, $\mathrm{S} / \mathrm{H}$ holds the inductor current value before reference tracking and this value is compared with 


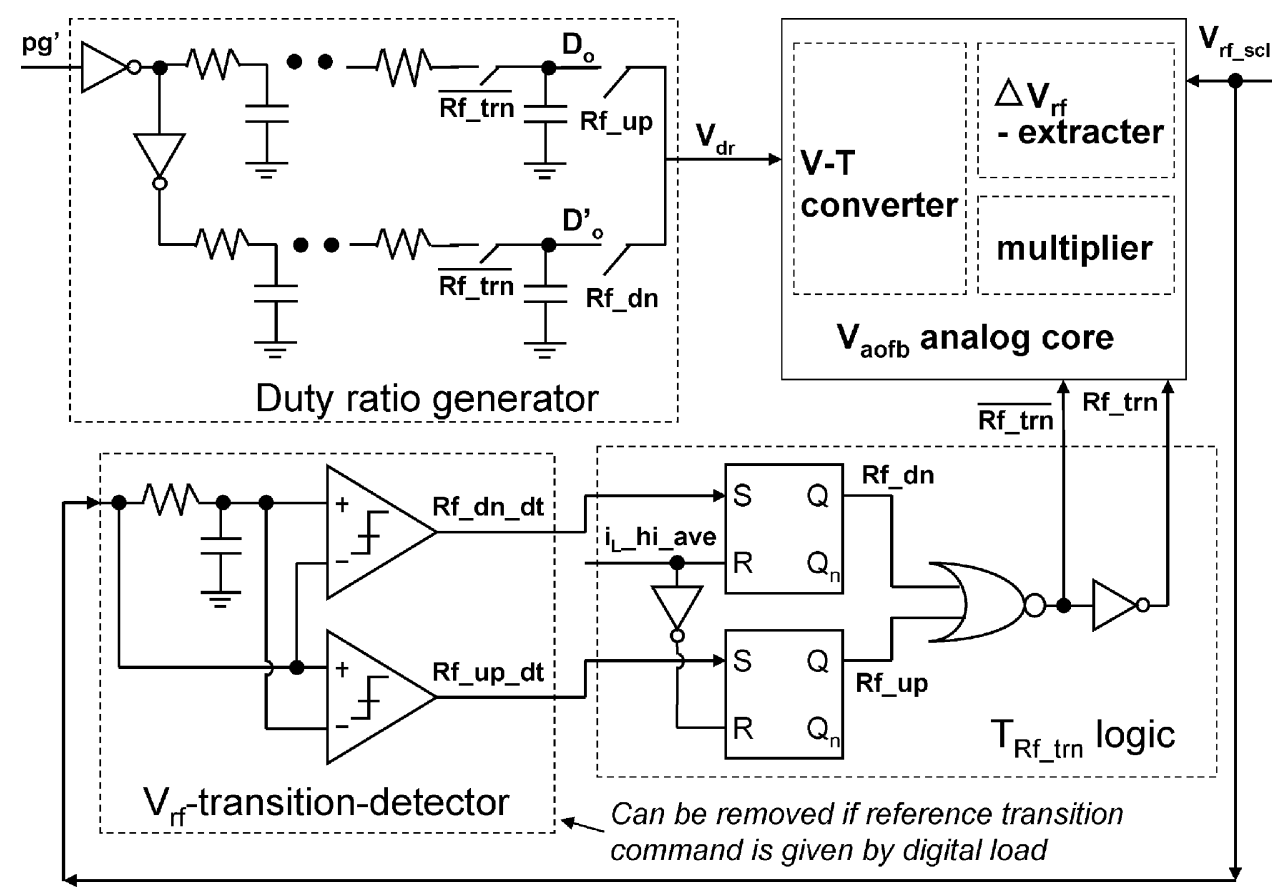

Fig. 9. Main components in AOFB-generator.

the contemporary inductor current to determine when $i_{L}$ returns to its original value. When this happens, it is signaled by " $i_{L}$-hi_ave" and its complement. The current sensors used are similar to a previous work [13] for its simplicity, accuracy, and ability to sense in the conduction periods of both power transistors. Both forward- and reverse- $i_{L}$ over-current protections are done (using signals " $i_{L}$ hi" and " $i_{L}$ low" in Fig. 7) since large forward and reverse inductor currents are necessary for fast reference tracking.

Duty-ratio generator generates duty-ratio $D$ and its complement $1-D$, which are used in reference down- and up-tracking, respectively. They are obtained by two sets of cascaded 4-stage low-pass filter acting on the pre-buffered switch gate-drive signal pg'. The outputs are then selected by Rf_dn and Rf_up.

The analog core of AOFB-scheme is shown in Fig. 10. The multiplier of duty-ratio and reference step size is a simple switched-capacitor circuit making use of the principle that charge being the product of current and time. Hence, the $\Delta V_{\mathrm{rf}}$-extractor is a $\mathrm{V}$-I converter that outputs constant current, which is proportional to $\Delta V_{\text {rf_scl }_{-}}$; while duty-ratio is translated to time by a voltage-to-time converter (V-T converter). Their operations are explained as follows.

In steady-state, M01-M06 in $\Delta V_{\mathrm{rf}}$-extractor conducts current of $V_{\text {rf_scl }} / R \Delta_{\mathrm{vrf}}$ so that $C_{\mathrm{hd}}$ samples information of $V_{\mathrm{rf} \_s c l}$. At $t_{0}$ when Rf_trn turns high, switch sw1 opens and original $V_{\text {rf_scl }}$ information is held in $C_{\text {hd }}$. Then, M03 keeps sourcing previous current while M06 sinks current corresponding to new $V_{\text {rf_scl. }}$ Difference between these two currents is injected into $C_{\text {aofb }}$ in multiplier via $\mathrm{T}_{\text {mult }}$ switch when $\mathrm{T}_{\text {mult }}$ remains high. $\mathrm{T}_{\text {mult }}$ is modulated by $\mathrm{V}-\mathrm{T}$ converter such that it is a pulse that starts when Rf_trn goes high, and its duration is directly proportional to the output of the duty-ratio generator, $V_{\mathrm{dr}}$. The multiplier's output $\left(V_{\mathrm{aofb}}\right)$ is reset to vl in steady-state, and it only performs multiplication when Rf_trn is high. It can be proved that after the $\mathrm{T}_{\text {mult }}$ pulse, change of $V_{\mathrm{aofb}}$ is given by

$$
\Delta V_{\mathrm{aofb}}=\frac{b \Delta V_{\mathrm{o}} V_{i} D C_{\mathrm{Tmult}}}{R_{\Delta \mathrm{vrf}} C_{\mathrm{aofb}} I_{k}} \propto D \Delta V_{0} .
$$

Thus, the circuit implements AOFB-scheme, in which parameters $V_{i}, C_{\mathrm{aofb}}, C_{\text {Tmult }}, R \Delta_{\mathrm{vrf}}$, and $\mathrm{I}_{k}$ determine $k$ in (7). Meanwhile, in this design the duration of reference tracking (i.e., $\left.t_{1}+t_{2}\right)$ is around a few microseconds, while the time to the optimum switching time $\left(t_{1}\right)$ can be less than two microseconds. Hence, another constraint of design is $\mathrm{T}_{\text {mult }}<0.5 \mu$ s so that multiplication ends before switching time $t_{1}$. At $t_{1}+t_{2}$ when Rf_trn turns low, the process of reference tracking is over, and $V_{\text {aofb }}$ is reset to vl. The addition of $V_{\text {aofb }}$ to $V_{\text {pwm_rmp }}$ is via voltage-to-current converter (similar to [12]) with one input being $V_{\text {aofb }}$ and another input being vl. Thus, both positive and negative $\Delta V_{\text {aofb }}$ additions can be done which are required in reference up- and down-tracking, respectively. All of the AOFB circuits, including current sensors, are integrated on-chip.

\section{EXPERIMENTAL RESULTS}

The proposed buck converter was implemented in AMS $0.35 \mu \mathrm{m}$ CMOS process. Fig. 11 is the die photo of the prototype, which has a dimension of $1.65 \mu \mathrm{m} \times 1.1 \mu \mathrm{m}$, including pads. The prototype works with input voltages ranging from $1.8 \mathrm{~V}$ to $3.3 \mathrm{~V}$, while inductor and output capacitor chosen to be $4.7 \mu \mathrm{H}$ and $9.4 \mu \mathrm{F}$ (with $0.1 \Omega$; ESR), respectively. Unless otherwise stated, all of the following results are obtained using input voltage of $3 \mathrm{~V}$, and resistive load. Table II shows the static performance summary of the prototype; in particular, the tight load regulation of $1.25 \mathrm{mV} / \mathrm{A}$ is due to the high open-loop gain of the amplifier $(\sim 60 \mathrm{~dB})$; while about half of output ripple voltage $(\sim 18 \mathrm{mV})$ is due to equivalent series inductance (ESL) 

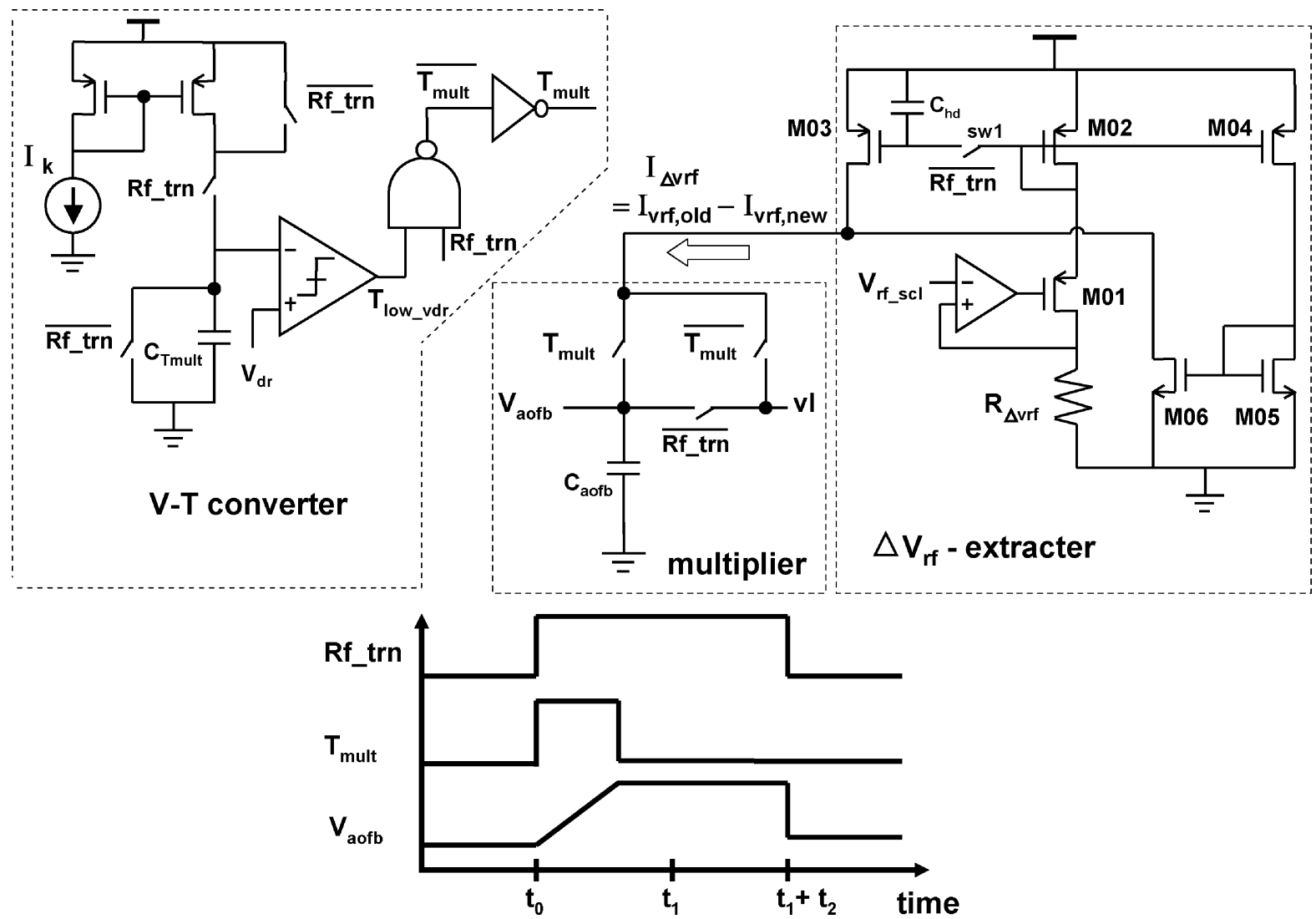

Fig. 10. Schematic and timing diagram of analog core of AOFB-generator.

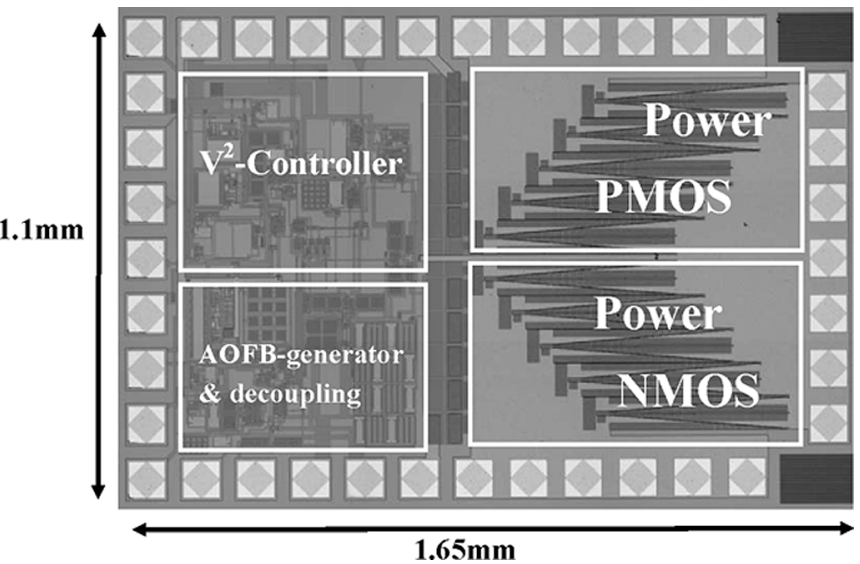

Fig. 11. Die photo of proposed $\mathrm{V}^{2}$-controlled buck converter.

on PCB. Fig. 12 shows the measured efficiency, which has a maximum of $96 \%$ at $260 \mathrm{~mA}$ load current.

Fig. 13 shows the measured load transient. At $V_{\mathrm{o}}=1.8 \mathrm{~V}$, a load current step of $-700 \mathrm{~mA}$ can be settled in only $3 \mu \mathrm{s}$. The clock-holding function is verified as it is noted that Hld_clk stays high during $V_{\mathrm{o}}$ slewing down; and as soon as it turns low, a clock pulse is issued so that $i_{L}$ increases instantly without delay. The response of a positive load current step is similarly fast, so it is not shown here for limited space.

Fig. 14 shows the measured reference up-tracking of $\mathrm{V}^{2}$ architecture with EPP. Undesirable effects including overshoot

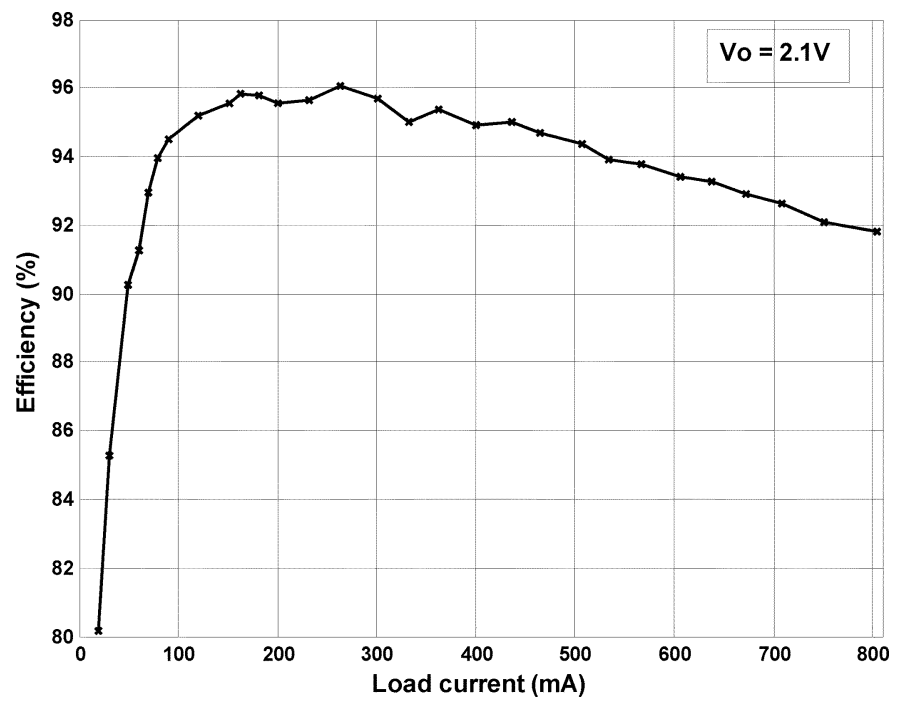

Fig. 12. Measured power conversion efficiency of the prototype.

of $V_{\mathrm{o}}$, too large peak $i_{L}$ and its subsequent undershoot are seen. As a comparison, Fig. 15 shows the case when AOFB-scheme is employed. The addition and removal of $V_{\text {aofb }}$ on $V_{\text {pwm_rmp }}$ is clearly seen on the buffered $V_{\text {pwm_rmp }}$ test signal. As a result, all of the above undesirable effects are removed and the benefits of lower transition energy and an ultra-fast transition time, $t_{\mathrm{rf} \_\mathrm{tk}}$, of $4 \mu \mathrm{s}$ are achieved. The comparison in reference 
TABLE II

Summary of StATIC Performance of the Prototype

\begin{tabular}{c|c}
\hline Technology & AMS $0.35-\mu \mathrm{m}$ CMOS process \\
\hline Switching frequency & $1 \mathrm{MHz}$ \\
\hline Input voltage & $1.8 \mathrm{~V}$ to $3.3 \mathrm{~V}$ (nominal 3V) \\
\hline Maximum output current & $800 \mathrm{~mA}$ \\
\hline Power conversion efficiency & max. $96 \% @ \mathrm{~V}_{\mathrm{o}}=2.1 \mathrm{~V}$ \\
\hline Line regulation & $9 \mathrm{mV} / \mathrm{V} @ \mathrm{~V}_{\mathrm{o}}=1.5 \mathrm{~V}$ \\
\hline Load regulation & $1.25 \mathrm{mV} / \mathrm{A}$ \\
\hline Output voltage range & $0.7 \mathrm{~V}-2.4 \mathrm{~V}$ \\
\hline Output ripple voltage & $35 \mathrm{mV} @ \mathrm{~V}_{\mathrm{o}}=1.8 \mathrm{~V}$ \\
\hline Chip area & $1.65 \mathrm{~mm} \times 1.1 \mathrm{~mm}$ (including pads) \\
\hline
\end{tabular}

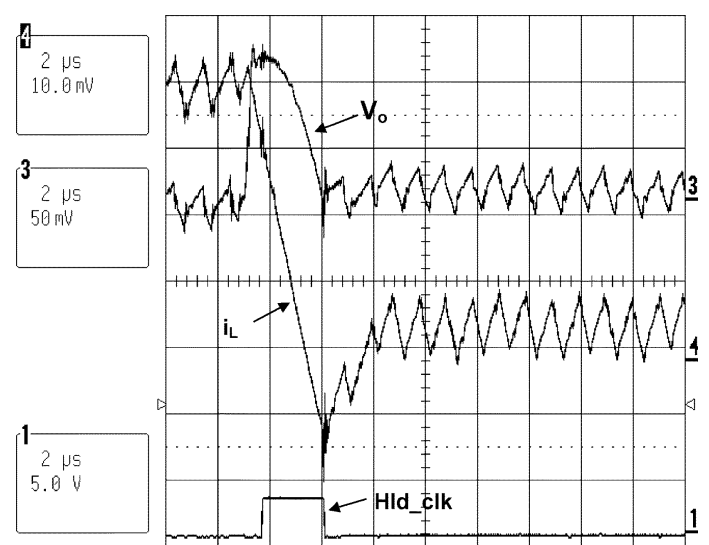

Fig. 13. Measured load transient $\left(V_{\mathrm{o}}=1.8 \mathrm{~V}\right.$, load current from $760 \mathrm{~mA}$ to $60 \mathrm{~mA})$.

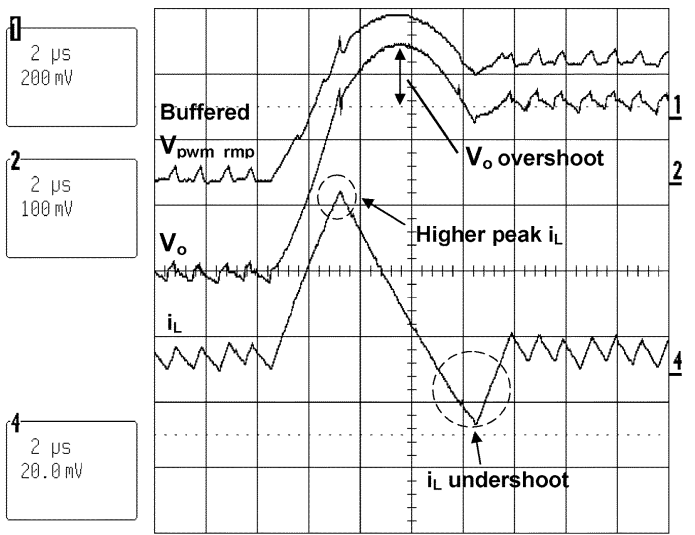

Fig. 14. Measured reference up-tracking of $\mathrm{V}^{2}$ with EPP only $\left(V_{\mathrm{o}}: 0.9 \mathrm{~V}->\right.$ $1.17 \mathrm{~V})$

down-tracking between $\mathrm{V}^{2}$-control with and without AOFB is similar and is not detailed here for brevity.

Figs. 16-19 shows the measured reference tracking of AOFB- $V^{2}$-architecture under different $V_{\mathrm{o}}$ s (i.e., different $D \mathrm{~s})$ and $\Delta V_{\mathrm{o}} \mathrm{s}$ using the worst case $k$-value as mentioned in Section IV. As expected, no undershoot/ overshoot of $V_{\mathrm{o}}$ is seen and the over-designed $k$-value only slows down the non-worst-case (i.e., smaller $\Delta V_{\mathrm{o}}$ or lower $D$ in reference down-tracking/higher $D$ in reference up-tracking) a little bit. In all cases, reference tracking finishes in $5 \mu$ s for steps as large

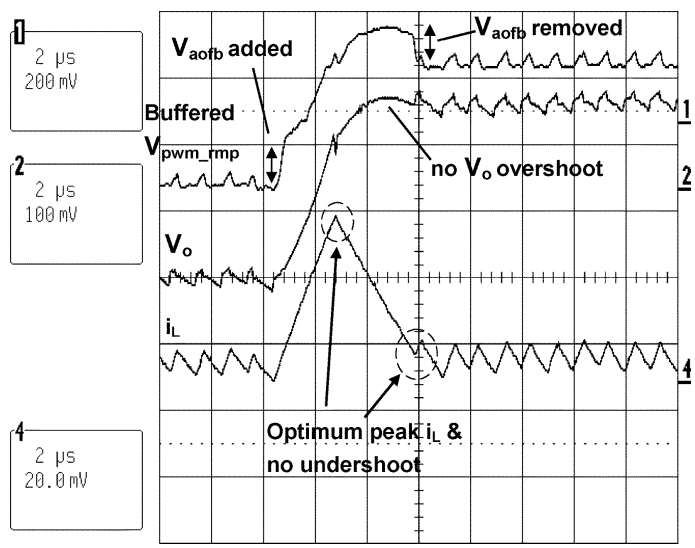

Fig. 15. Measured reference up-tracking of $\mathrm{V}^{2}$ with EPP \& AOFB $\left(V_{\mathrm{o}}\right.$ : $0.9 \mathrm{~V}->1.17 \mathrm{~V})$.

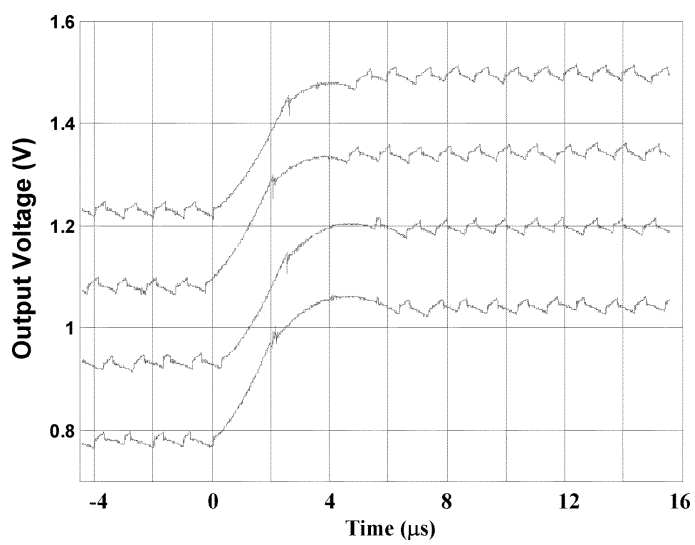

Fig. 16. Measured reference up-tracking (same $\Delta V_{\mathrm{o}}$, different $D \mathrm{~s}$ ).

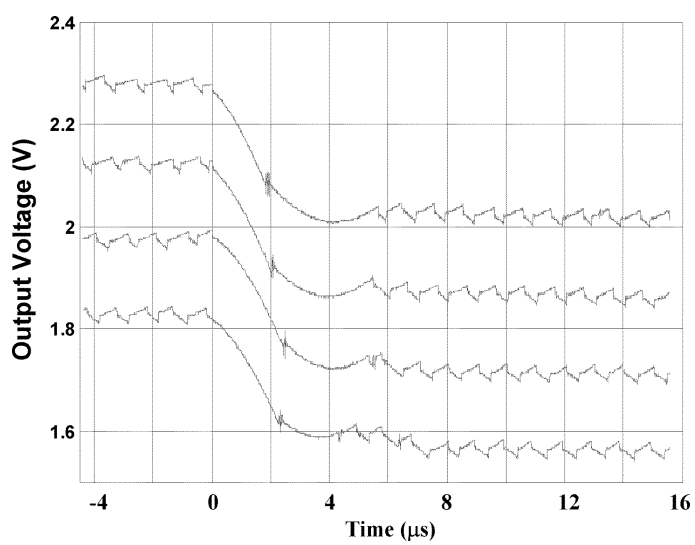

Fig. 17. Measured reference down-tracking (same $\Delta V_{\mathrm{o}}$, different $D \mathrm{~s}$ ).

as $500 \mathrm{mV}$. Larger step requires higher peak $i_{L}$ exceeding the over-current limit, which turns the optimum near-triangular $i_{L}$ profile into a chopped one. As this is not the case of optimum reference tracking, the result is not shown here. Yet, the prototype still undergoes larger step reference tracking with a sub-optimum speed limited by maximum $i_{L}$.

Table III shows the comparison of this work and some prior adaptive converters. Typically, the figure-of-merit, $t_{\mathrm{rf}-\mathrm{tk}} / \Delta V_{\mathrm{o}}$, is used as a measure of tracking speed. Yet, as shown in Section IV, optimum tracking time scales with $\sqrt{L C_{0}}$, which 
TABLE III

COMPARISON OF ADAPTIVE SWITCHING CONVERTERS.

\begin{tabular}{|c|c|c|c|c|c|c|}
\hline Design & {$[4]$} & {$[14]$} & {$[15]$} & {$[16]$} & {$[17]$} & $\begin{array}{c}\text { This } \\
\text { Work }\end{array}$ \\
\hline FOM $_{\text {conventional }}(\mu \mathrm{s} / \mathrm{V})$ & 26.92 & 292 & 80 & 12.25 & 41.67 & 10 \\
\hline FOM $_{\text {ctl }}(1 / \sqrt{\mathrm{V}})$ & 8.54 & 14.6 & 4.42 & $2.73 *$ & 35.64 & 1.06 \\
\hline Output range $(\mathrm{V})$ & $1.2-3.8$ & $0-0.5$ & $1.1-2.3$ & $0.9-2.5$ & $1.3-1.9$ & $0.7-2.4$ \\
\hline Max efficiency $(\%)$ & 90 & 92 & 95 & 93.7 & N/A & 96 \\
\hline CMOS process $(\mu \mathrm{m})$ & 0.6 & $0.35 \mathrm{SOI}$ & 0.25 & 0.5 & N/A & 0.35 \\
\hline Area $\left(\mathrm{mm}^{2}\right)$ & 5.44 & 6 & 1.43 & 2.31 & N/A & 1.815 \\
\hline
\end{tabular}

$* \mathrm{~L}, \mathrm{C}_{\mathrm{o}}$ estimated from steady-state ripple voltage and current

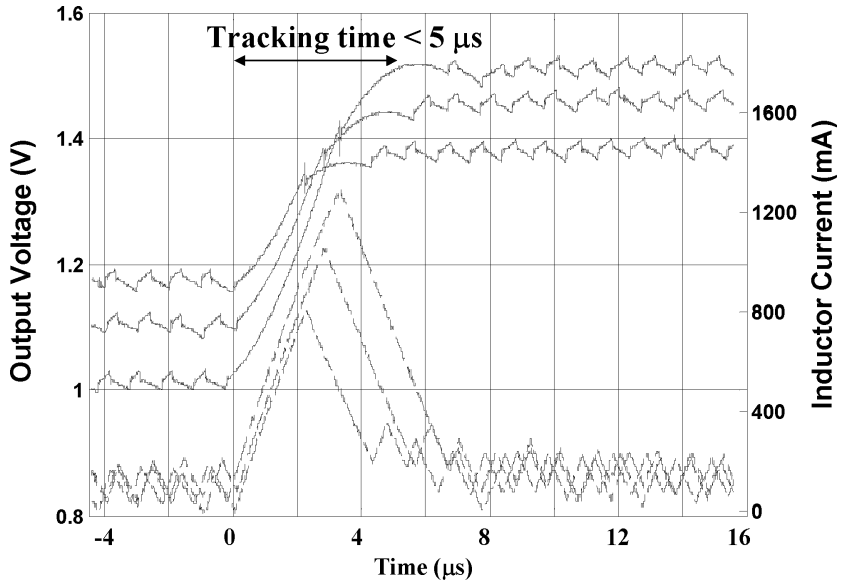

Fig. 18. Measured reference up-tracking (different $\Delta V_{\mathrm{o}} \mathrm{s}$, same $D$ ).

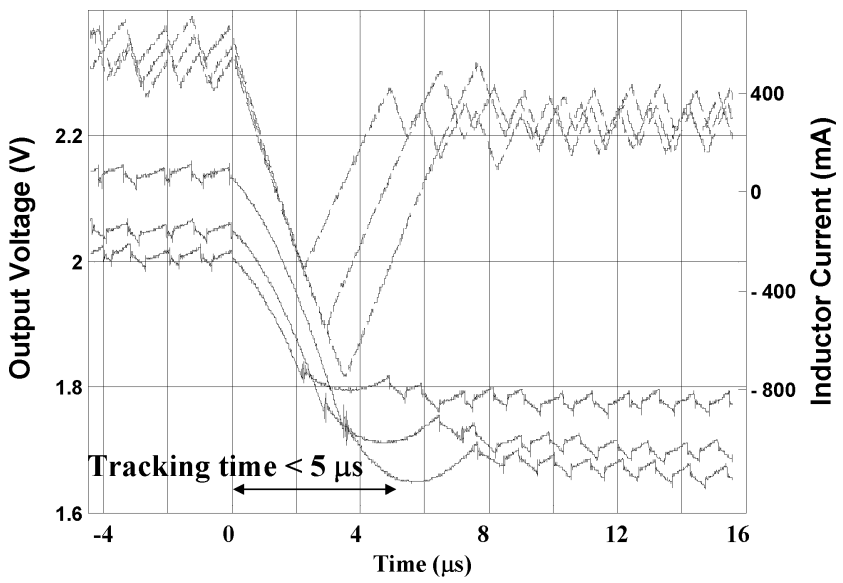

Fig. 19. Measured reference down-tracking (different $\Delta V_{\mathrm{o}} \mathrm{s}$, same $D$ ).

is an indicator of the power stage's speed and $\sqrt{\Delta V_{\mathrm{o}}}$, but not $\Delta V_{\mathrm{o}}$. Hence, we propose a new figure-of-merit to reflect the actual reference tracking speed of the controller:

$$
\mathrm{FOM}_{\mathrm{ctl}}=\frac{t_{\mathrm{rf} \_\mathrm{tk}}}{\sqrt{\Delta V_{\mathrm{o}} L C_{\mathrm{o}}}}
$$

It should be noted that the unit of $\mathrm{FOM}_{\mathrm{ctl}}$ is $1 / \sqrt{\mathrm{V}}$ as tracking time is normalized by the time constant of power stage. The proposed AOFB- $\mathrm{V}^{2}$-architecture achieves the lowest $\mathrm{FOM}_{\mathrm{ctl}}$, i.e., the fastest transition speed of the controller compared to prior arts.

\section{CONCLUSION}

A high-performance $\mathrm{V}^{2}$-controlled buck converter suitable for DVS applications is presented and proven by experimental results. In addition to the original merits of $\mathrm{V}^{2}$-control including easy compensation, fast load step response, tight regulation, and fixed switching frequency, performance metrics important for DVS systems like fast tracking time, low switching loss and conduction loss during reference tracking are also achieved thanks to the employment of end-point prediction, a novel oscillator and the proposed AOFB-scheme. The dynamics of reference tracking is analyzed, and the criterion for optimum reference tracking is introduced, which can also be extended to other output-ripple-based converters such as hysteretic control.

\section{REFERENCES}

[1] V. Gutnik and A. P. Chandrakasan, "Embedded power supply for lowpower DSP," IEEE Trans. Very Large Scale Integr. (VLSI) Syst., vol. 5 , no. 4, pp. 425-435, Dec. 1997.

[2] T. Kuroda, K. Suzuki, S. Mita, T. Fujita, F. Yamane, F. Sano, A. Chiba, Y. Watanabe, K. Matsuda, T. Maeda, T. Sakurai, and T. Furuyama, "Variable supply-voltage scheme for low-power high-speed CMOS digital design," IEEE J. Solid-State Circuits, vol. 33, no. 3, pp. 454-462, Mar. 1998.

[3] G. Wei, J. Kim, D. Liu, S. Sidiropoulos, and M. Horowitz, "A variable-frequency parallel I/O interface with adaptive power supply regulation," in IEEE ISSCC Dig. Tech. Papers, 2000, pp. 298-299.

[4] T. D. Burd, T. A. Pering, A. J. Stratakos, and R. W. Brodersen, "A dynamic voltage scaled microprocessor system," IEEE J. Solid-State Circuits, vol. 35, no. 11, pp. 1571-1580, Nov. 2000.

[5] S. Dhar and D. Maksimovic, "Switching regulator with dynamically adjustable supply voltage for low power VLSI," in Proc. 27th Annu. Conf. IEEE Industrial Electronics Soc. (IECON'01), Denver, CO, 2001, vol. 3, pp. 1874-1879.

[6] A. Soto, A. de Castro, P. Alou, J. A. Cobos, J. Uceda, and A. Lotfi, "Analysis of the buck converter for scaling the supply voltage of digital circuits," in Proc. 18th Annu. Applied Power Electronics Conf. (APEC 2003), Miami Beach, FL, 2003, vol. 2, pp. 711-717.

[7] A. Soto, P. Alou, and J. A. Cobos, "Nonlinear digital control breaks bandwidth limitations," in Proc. 21st Annu. IEEE Applied Power Electronics Conf. (APEC'O6), Mar. 2006, pp. 724-730.

[8] D. Gorder and W. R. Pelletier, "V" ${ }^{2}$ architecture provides ultra fast transient response in switch mode power supplies," in Proc. High Frequency Power Conversion Conf. (HFPC'96), 1996, pp. 19-23.

[9] S. Qu, "Modeling and design considerations of $\mathrm{V}^{2}$ controlled buck regulator," in Proc. IEEE Applied Power Electronics Conf. (APEC'01), Mar. 2001, pp. 507-513.

[10] NCV8800 Series, Synchronous Buck Regulator with 1.0 Amp Switch. On Semiconductor, Phoenix, AZ [Online]. Available: http://www.onsemi.com/pub/Collateral/NCV8800-D.PDF

[11] M. Siu, P. K. T. Mok, K. N. Leung, Y.H. Lam, and W. H. Ki, "A voltagemode PWM buck regulator with end-point prediction," IEEE Trans. Circuits Syst. II, Expr. Briefs, vol. 53, no. 4, pp. 294-298, Apr. 2006.

[12] C. Lee and P. Mok, "A monolithic current-mode CMOS DC-DC converter with on-chip current-sensing technique," IEEE J. Solid-State Circuits, vol. 39, no. 1, pp. 3-14, Jan. 2004. 
[13] Y. H. Lam, W. H. Ki, C. Y. Tsui, and D. Ma, "Integrated 0.9 V chargecontrol switching converter with self-biased current sensor," in Proc. IEEE Int. Midwest Symp. Circuits and Systems, 2004, pp. 305-308.

[14] T. Fuse, A. Kameyama, M. Ohta, and K. Ohuchi, "A $0.5 \mathrm{~V}$ power-supply scheme for low power LSI's using multi-Vt SOI CMOS technology," in Symp. VLSI Circuits Dig. Tech. Papers, Jun. 2001, pp. 219-220.

[15] J. Kim and M. A. Horowitz, "An efficient digital sliding controller for adaptive power-supply regulation," IEEE J. Solid-State Circuits, vol. 37, no. 5, pp. 639-647, May 2002.

[16] D. Ma, W. H. Ki, and C. Y. Tsui, "An integrated one-cycle control buck converter with adaptive output and dual loops for output error correction," IEEE J. Solid-State Circuits, vol. 39, no. 1, pp. 140-149, Jan. 2004

[17] G. Wei, O. Trecases, and W. T. Ng, "A dynamic voltage scaling controller for maximum energy saving across full range of load conditions," in Proc. 2005 IEEE Conf. Electron Devices and Solid-State Circuits, Hong Kong, 2005, pp. 371-374.

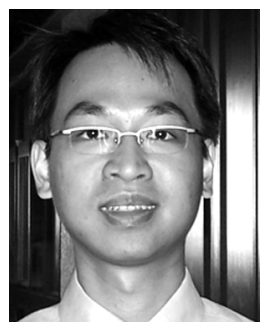

Patrick Y. Wu received the B.Eng. degree in electrical engineering from the University of Hong Kong in 2001, and the MSc. degree in electrical engineering from the University of Southern California, Los Angeles, in 2003. He is currently working towards the Ph.D. degree in electrical engineering at the Hong Kong University of Science and Technology (HKUST).

His research interests include design of data converter, switched-capacitor and switched-opamp circuits, switch mode power supply and its control

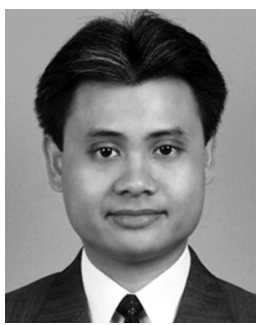

Philip K. T. Mok (S'86-M'95-SM'02) received the B.A.Sc., M.A.Sc., and Ph.D. degrees in electrical and computer engineering from the University of Toronto, Toronto, ON, Canada, in 1986, 1989 and 1995, respectively.

In January 1995, he joined the Department of Electronic and Computer Engineering, The Hong Kong University of Science and Technology, Hong Kong, China, where he is currently an Associate Professor. His research interests include semiconductor devices, processing technologies and circuit designs for power electronics and telecommunications applications, with current emphasis on power management integrated circuits, low-voltage analogue integrated circuits and RF integrated circuits design.

Dr. Mok received the Henry G. Acres Medal, the W.S. Wilson Medal and a Teaching Assistant Award from the University of Toronto, and the Teaching Excellence Appreciation Award twice from The Hong Kong University of Science and Technology. He is also a co-recipient of the Best Student Paper Award in the 2002 IEEE Custom Integrated Circuits Conference. In addition, he has been a member of the International Technical Program Committees of the IEEE International Solid-State Circuits Conference (ISSCC) since 2005 and he has served as an associate editor for IEEE TRANSACTIONS ON CIRCUITS AND SYSTEMS PART II and IEEE JOURNAL OF SOLID-STATE CIRCUITS since 2006. methodology. 